\title{
Dynamical Symmetry Breaking in Transport through Molecules
}

\author{
Andrea Donarini, Milena Grifoni, and Klaus Richter \\ Theoretische Physik, Universität Regensburg, 93040 Regensburg, Germany
}

(Received 4 May 2006; published 16 October 2006)

\begin{abstract}
We analyze the interplay between vibrational and electronic degrees of freedom in charge transport across a molecular single-electron transistor. We focus on the wide class of molecules which possess quasidegenerate vibrational eigenstates, while no degeneracy occurs for their anionic configuration. We show that the combined effect of a thermal environment and coupling to leads, involving tunneling events charging and discharging the molecule, leads to a dynamical symmetry breaking where quasidegenerate eigenstates acquire different occupations. This imbalance gives rise to a characteristic asymmetry of the current versus an applied gate voltage.
\end{abstract}

DOI: 10.1103/PhysRevLett.97.166801

PACS numbers: 85.65.+h, 73.63.-b, 85.85.+j

Molecular electronics is a promising answer to the demand of miniaturization, reproducibility, and flexibility of electronic devices. Starting with the pioneering work of Aviram and Ratner [1], and especially with the first singlemolecule measurement by Reed et al. [2], molecular electronics has become an active research field both experimentally and theoretically [3]. Still, fundamental questions on the peculiar nature of single-molecule junctions and their novel functionalities remain open. Among others they involve the unique electromechanical properties of molecular junctions which render these distinctly different from, e.g., semiconductor quantum dots.

Recent research on this particular topic has revealed a number of interesting effects such as shuttling instabilities [4-6], Franck-Condon blockade [7,8], and, more generally, conformational [9-11] or vibronic [12-15] signatures in the electron transport characteristics. In this respect, however, the role of coherences in transport due to quasidegenerate levels has not been highlighted.

In this Letter we consider charge transport through a molecular junction weakly contacted to leads. At low enough temperatures charging effects prevent that more than one excess electron at a time can populate the molecule. The weak coupling ensures that the potential drop between source and drain leads is concentrated in the contact region, and no substantial electric field acts directly on the molecule. We focus on the ubiquitous, but so far poorly investigated, case of adiabatic vibronic potentials with more than one stable configuration as, e.g., found in oligoparaphenylenes. In particular, we address molecules characterized by eigenstate dubletts in the neutral configuration and nondegenerate anionic states, or vice versa. This is sketched in Fig. 1, which can represent, e.g., the adiabatic potential energy surfaces of biphenyl-based molecules as a function of the dihedral angle (here playing the role of the $x$ coordinate) between the phenyl rings [15]. These molecules have been studied in recent transport experiments [16,17]. Specifically, in [17] for the first time quantitatively, the connection between the angular conformation and the electrical conductance has been proved for several biphenyl-based molecules.

We show that dynamical symmetry breaking (DSB), where quasidegenerate eigenstates are differently occupied, may occur and affect transport, while in the absence of couplings to the leads these states are equally populated at finite temperature, and hence the system does not prefer a definite parity. Temperature, on the other hand, should not exceed a critical value above which DSB is lost due to dephasing, caused by a thermal bath. Solving the master equation for the reduced density matrix including coherences, we demonstrate the possibility of detecting DSB in the current through the molecule under different bias and gating conditions.

To address transport in the single-electron regime, where only the electronic ground states $|0\rangle$ of the neutral and $|1\rangle$

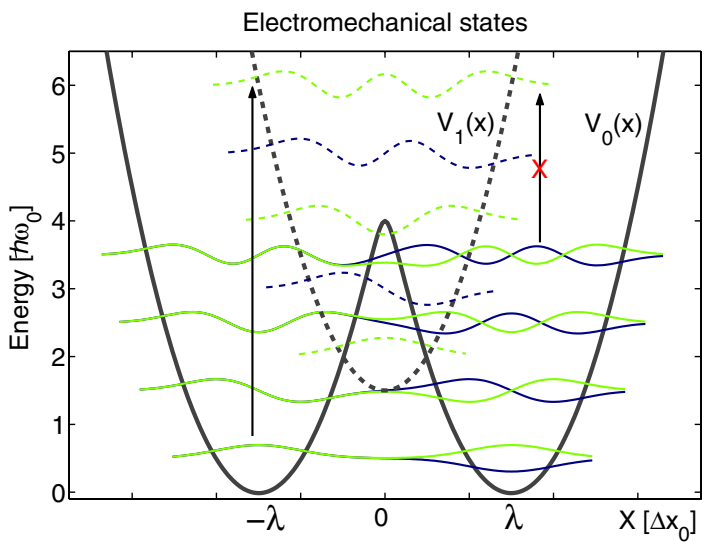

FIG. 1 (color online). Electromechanical states of the molecule. Thick black lines indicate the adiabatic potentials $V_{0}$ and $V_{1}$ for the neutral (solid) and singly charged (dashed) molecule. Thin green (blue) lines denote the even (odd) vibrational eigenfunctions (for the two potentials) displayed with a vertical shift equal to their corresponding energy eigenvalue. Energies are given in units of $\hbar \omega_{0}$ and lengths in terms of the zero-point motion $\Delta x_{0}=\sqrt{\hbar / m \omega_{0}}$. The arrows mark examples of allowed and forbidden transitions. 
of the anionic configuration are involved, we describe the molecule through the Hamiltonian

$$
H_{S}=\frac{p^{2}}{2 m}+|0\rangle\left\langle 0\left|V_{0}(x)+\right| 1\right\rangle\langle 1| V_{1}(x)
$$

in terms of the corresponding adiabatic potential energy surfaces $V_{0}(x), V_{1}(x)$ associated with the softest mode coordinate $x$. The adiabatic potentials and the related vibrational eigenfunctions are sketched in Fig. 1. The neutral molecule is characterized by the double well potential $V_{0}$ with minima located at $\pm \lambda$. For large enough barriers, i.e., exponentially small tunnel splitting, the low-lying eigenstates are organized in pairs of (quasi)degenerate wave functions. The anion is modeled by a single harmonic potential $V_{1}$ with frequency $\omega_{1}$, centered at the origin of the coordinate system (dashed line in Fig. 1).

We express the system coupled to leads and in contact with a thermal bath through the Hamiltonian

$$
H=H_{S}+H_{L}+H_{R}+H_{B}+V_{T}+V_{\mathrm{SB}} .
$$

The leads are described as reservoirs of noninteracting quasiparticles in terms of $H_{L}+H_{R}=\sum_{k ; \alpha=L, R}\left(\epsilon_{k \alpha}-\right.$ $\left.\mu_{\alpha}\right) c_{k \alpha}^{\dagger} c_{k \alpha}$, where $\mu_{L, R}= \pm \Delta V / 2$ accounts for the effects of a symmetrically applied bias voltage and $c_{k \alpha}^{\dagger}\left(c_{k \alpha}\right)$ creates (destroys) an electron in the lead $\alpha$. Transfer of electrons is mediated by the tunneling Hamiltonian $V_{T}=$ $v \sum_{k ; \alpha=L, R}\left(|0\rangle\left\langle 1\left|c_{k \alpha}^{\dagger}+\right| 1\right\rangle\langle 0| c_{k \alpha}\right)$. Finally, $H_{B}$ describes a thermal bath of harmonic oscillators coupled linearly to the displacement coordinate $x$ via the system-bath Hamiltonian $V_{\mathrm{SB}}=\hbar \tilde{g} x \sum_{q}\left(d_{q}^{\dagger}+d_{q}\right)$, where $d_{q}^{\dagger}$ and $d_{q}$ are bosonic creation and destruction operators associated with the oscillator with energy $\hbar \omega_{q}$.

Since we are interested only in the low-energy states, we approximate $V_{0}(x)$ by two harmonic wells centered at $\pm \lambda$ and with frequency $\omega_{0}$. Formally, this is achieved by projecting the Hamiltonian $H$ on a smaller Hilbert space in which we can define the identity operator as

$$
\mathbb{1}=|1\rangle\langle 1|+| 0\rangle\langle 0|\left(\mathcal{P}_{+}+\mathcal{P}_{-}\right) .
$$

Here $\mathcal{P}_{ \pm}=\sum_{n=0}^{N}|n, \pm\rangle\langle n, \pm|$ are projection operators on the states right or left to the barrier. They are defined in terms of the shifted harmonic oscillator eigenvectors $|n, \pm\rangle \equiv \exp (\mp i \lambda p / \hbar)|n\rangle$. The condition of negligible overlap between these eigenfunctions reads $\mathcal{P}_{+} \mathcal{P}_{-}=$ $\mathcal{P}_{-} \mathcal{P}_{+}=0$. In the Hilbert space defined by identity (3) we obtain as effective system Hamiltonian

$$
\begin{aligned}
H_{S}^{\mathrm{eff}}= & |0\rangle\langle 0| \sum_{\tau= \pm}\left[\mathcal{P}_{\tau}\left(\frac{1}{2}+d_{\tau}^{\dagger} d_{\tau}\right) \mathcal{P}_{\tau}\right] \hbar \omega_{0} \\
& +|1\rangle\langle 1|\left[\left(\frac{1}{2}+d^{\dagger} d\right) \hbar \omega_{1}+e V_{g}-\epsilon_{a}\right],
\end{aligned}
$$

where $d_{ \pm}^{\dagger}$ and $d^{\dagger}$ are operators for the neutral and charged configurations, $V_{g}$ is the gate voltage, and $\epsilon_{a}$ the electron affinity. Along similar lines we find for the effective tunneling and system-bath Hamiltonians

$$
\begin{aligned}
V_{T}^{\mathrm{eff}}= & v \sum_{k ; \alpha=L, R}\left[|0\rangle\langle 1| c_{k \alpha}^{\dagger}\left(\mathcal{P}_{+}+\mathcal{P}_{-}\right)+\text {H.c. }\right], \\
V_{\mathrm{SB}}^{\mathrm{eff}}= & \hbar g \sum_{q}\left(d_{q}+d_{q}^{\dagger}\right)\left\{\delta|1\rangle\langle 1|\left(d+d^{\dagger}\right)\right. \\
& \left.+|0\rangle\langle 0| \sum_{\tau= \pm}\left[\mathcal{P}_{\tau}\left(d_{\tau}+d_{\tau}^{\dagger}+\tau 2 \lambda\right) \mathcal{P}_{\tau}\right]\right\},
\end{aligned}
$$

with $g=\tilde{g} \sqrt{2 \hbar /\left(m \omega_{0}\right)}$ and $\delta=\sqrt{\omega_{1} / \omega_{0}}$. For transport, we seek stationary solutions of the Liouville equation

$$
\dot{\sigma}(t)=-\frac{i}{\hbar} \operatorname{Tr}_{\text {leads }+ \text { bath }}\left\{\left[H^{\text {eff }}, W(t)\right]\right\}
$$

for the reduced density matrix (RDM) $\sigma(t):=$ $\operatorname{Tr}_{\text {leads }+ \text { bath }}\{W(t)\}$, where $W(t)$ is the total density matrix associated with the effective Hamiltonian corresponding to that in Eq. (2). Treating the interactions $V_{T}^{\text {eff }}$ and $V_{\mathrm{SB}}^{\text {eff }}$ as perturbations, we rewrite Eq. (6) in terms of three contributions: $\dot{\sigma}(t)=\mathcal{L} \sigma(t)=\left(\mathcal{L}_{\text {coh }}+\mathcal{L}_{\text {tun }}+\mathcal{L}_{\text {damp }}\right) \sigma(t)$. The coherent part of the differential equation has the usual form $\mathcal{L}_{\text {coh }} \sigma(t)=-(i / \hbar)\left[H_{S}^{\text {eff }}, \sigma(t)\right]$ describing the evolution of the isolated molecule. The coupling to the leads/bath gives rise to the driving/damping terms $\mathcal{L}_{\text {tun }} \sigma(t)$ and $\mathcal{L}_{\text {damp }} \sigma(t)$.

We now perform the following standard approximations: we treat the leads as thermal reservoirs at equilibrium temperature $T$, consider the coupling to the leads up to second order in $V_{T}^{\text {eff }}$, and, being interested in the stationary solution, neglect non-Markovian contributions to the equation of motion. Furthermore, neglecting off-diagonal elements of the RDM between anionic and neutral configurations, as well as between nondegenerate eigenstates, and disregarding nonenergy conserving contributions, the anionic and neutral-state components read

$$
\begin{aligned}
\left(\mathcal{L}_{\text {tun }} \sigma\right)_{11}= & \sum_{\alpha=L, R ; \tau= \pm}\left[2\left(\Gamma_{\mathrm{in}}^{\alpha} \sigma_{00} \mathcal{P}_{\tau}+\mathcal{P}_{\tau} \sigma_{00} \Gamma_{\mathrm{in}}^{\alpha \dagger}\right)\right. \\
& \left.-\left(\mathcal{P}_{\tau} \Gamma_{\text {out }}^{\alpha} \sigma_{11}+\sigma_{11} \Gamma_{\text {out }}^{\alpha \dagger} \mathcal{P}_{\tau}\right)\right] \\
\left(\mathcal{L}_{\text {tun }} \sigma\right)_{00}^{\tau \tau^{\prime}}= & \sum_{\alpha=L, R} \mathcal{P}_{\tau}\left[\Gamma_{\text {out }}^{\alpha} \sigma_{11}+\sigma_{11} \Gamma_{\text {out }}^{\alpha \dagger}\right. \\
& \left.-2\left(\Gamma_{\text {in }}^{\alpha} \sigma_{00}+\sigma_{00} \Gamma_{\text {in }}^{\alpha \dagger}\right)\right] \mathcal{P}_{\tau^{\prime}}
\end{aligned}
$$

with rate matrices for tunneling into/out of the molecule

$$
\begin{aligned}
\Gamma_{\mathrm{in}}^{\alpha} & =\frac{\Gamma_{\alpha}}{2} \sum_{m, n, \tau= \pm}|m\rangle\langle n, \tau| f\left(e V_{g}+\epsilon_{n m}\right)\langle m \mid n, \tau\rangle, \\
\Gamma_{\text {out }}^{\alpha} & =\frac{\Gamma_{\alpha}}{2} \sum_{m, n, \tau= \pm}|n, \tau\rangle\langle m|-\Gamma_{\text {in }}^{\alpha \dagger} .
\end{aligned}
$$

Here $f(\epsilon)$ is the Fermi function, and $\Gamma_{\alpha}=(2 \pi / \hbar) D_{\alpha}|v|^{2}$ is the bare electronic rate, with $D_{\alpha}$ the density of states in lead $\alpha$ at the Fermi energy. For convenience we assumed equal frequencies $\omega_{0}=\omega_{1}$ of the neutral and anionic potential, and hence $\epsilon_{n m}=\hbar \omega_{0}(m-n)$; calculations for 
$\omega_{0} \neq \omega_{1}$ yielded no qualitative changes. Furthermore, we shifted the electrochemical potential $e V_{g}-\epsilon_{a} \rightarrow e V_{g}$ in the argument of the Fermi function. The overlap matrix elements $\langle m \mid n, \tau\rangle$ of the vibrational states of the neutral and charged configurations, known as Franck-Condon factors, determine, together with energy resonance conditions, the transition rates (8) and thus the transport characteristics of the junction. While the Franck-Condon factors are fixed by the adiabatic potentials, the bias and gate voltages influence the resonance conditions.

To describe relaxation and dephasing, we consider the case of an Ohmic bath with spectral density $J(\omega)=m \gamma \omega$, where $\gamma$ is the damping coefficient [18]. Along the same lines as for the tunneling term we find

$$
\begin{aligned}
\left(\mathcal{L}_{\mathrm{damp}} \sigma\right)_{11}= & -\frac{i \gamma}{2 \hbar}\left[x,\left\{p, \sigma_{11}\right\}\right] \\
& -\frac{\gamma m \omega_{0}}{\hbar}\left(\bar{N}+\frac{1}{2}\right)\left[x,\left[x, \sigma_{11}\right]\right], \\
\left(\mathcal{L}_{\mathrm{damp}} \sigma\right)_{00}^{\tau \tau^{\prime}}= & -\frac{i \gamma}{2 \hbar} \mathcal{P}_{\tau}\left[x,\left\{p, \sigma_{00}^{\tau \tau^{\prime}}\right\}\right] \mathcal{P}_{\tau^{\prime}} \\
& -\frac{\gamma m \omega_{0}}{\hbar}\left(\bar{N}+\frac{1}{2}\right) \mathcal{P}_{\tau}\left[x,\left[x, \sigma_{00}^{\tau \tau^{\prime}}\right]\right] \mathcal{P}_{\tau^{\prime}} \\
& -8 \gamma \frac{k_{B} T}{\hbar \omega_{0}} \lambda^{2}\left(\mathcal{P}_{+} \sigma_{00}^{\tau \tau^{\prime}} \mathcal{P}_{-}+\mathcal{P}_{-} \sigma_{00}^{\tau \tau^{\prime}} \mathcal{P}_{+}\right),
\end{aligned}
$$

where $\bar{N}(\omega)$ is the Bose distribution function. Note the occurrence of a term responsible for pure interwell dephasing and proportional to $T$ and $\lambda^{2}$ in the expression for $\left(\mathcal{L}_{\mathrm{damp}} \sigma\right)_{00}$.

Since we are interested in the long-time properties, we look for solutions of the stationary problem $\mathcal{L} \sigma^{\text {stat }}=0$. Given the stationary RDM $\sigma^{\text {stat }}$ we calculate the stationary current as the trace over the system degrees of freedom of the left or right current operators, $I^{\text {stat }}=\operatorname{Tr}_{\text {sys }}\left[\sigma^{\text {stat }} \hat{I}_{L}\right]=$ $\operatorname{Tr}_{\text {sys }}\left[\sigma^{\text {stat }} \hat{I}_{R}\right]$, with, e.g.,

$$
\begin{aligned}
\hat{I}_{L}= & \sum_{\tau= \pm}\left[2|0\rangle\langle 0|\left(\mathcal{P}_{\tau} \Gamma_{\text {in }}^{L}+\Gamma_{\text {in }}^{L \dagger} \mathcal{P}_{\tau}\right)\right. \\
& \left.-|1\rangle\langle 1|\left(\mathcal{P}_{\tau} \Gamma_{\text {out }}^{L}+\Gamma_{\text {out }}^{L \dagger} \mathcal{P}_{\tau}\right)\right] .
\end{aligned}
$$

We stress that not only populations but also coherences of the RDM in the ( \pm ) basis contribute to $I^{\text {stat }}$.

In the first column of the left side of Fig. 2 we present the results of our calculation of the gate- and bias-dependent current for decreasing temperature (from bottom to top). Besides the evidence of a Franck-Condon blockade [7,8] (where due to an exponential suppression of the FranckCondon factors $\langle m \mid n, \tau\rangle$ transport is blocked and the Coulomb diamonds no longer close) and current steps due to phononic excitations, Fig. 2 shows an increasing asymmetry in the gate voltage $V_{g}$ with decreasing temperature. To understand the origin of this asymmetry, we depict in the second and third column of the left side of Fig. 2 the
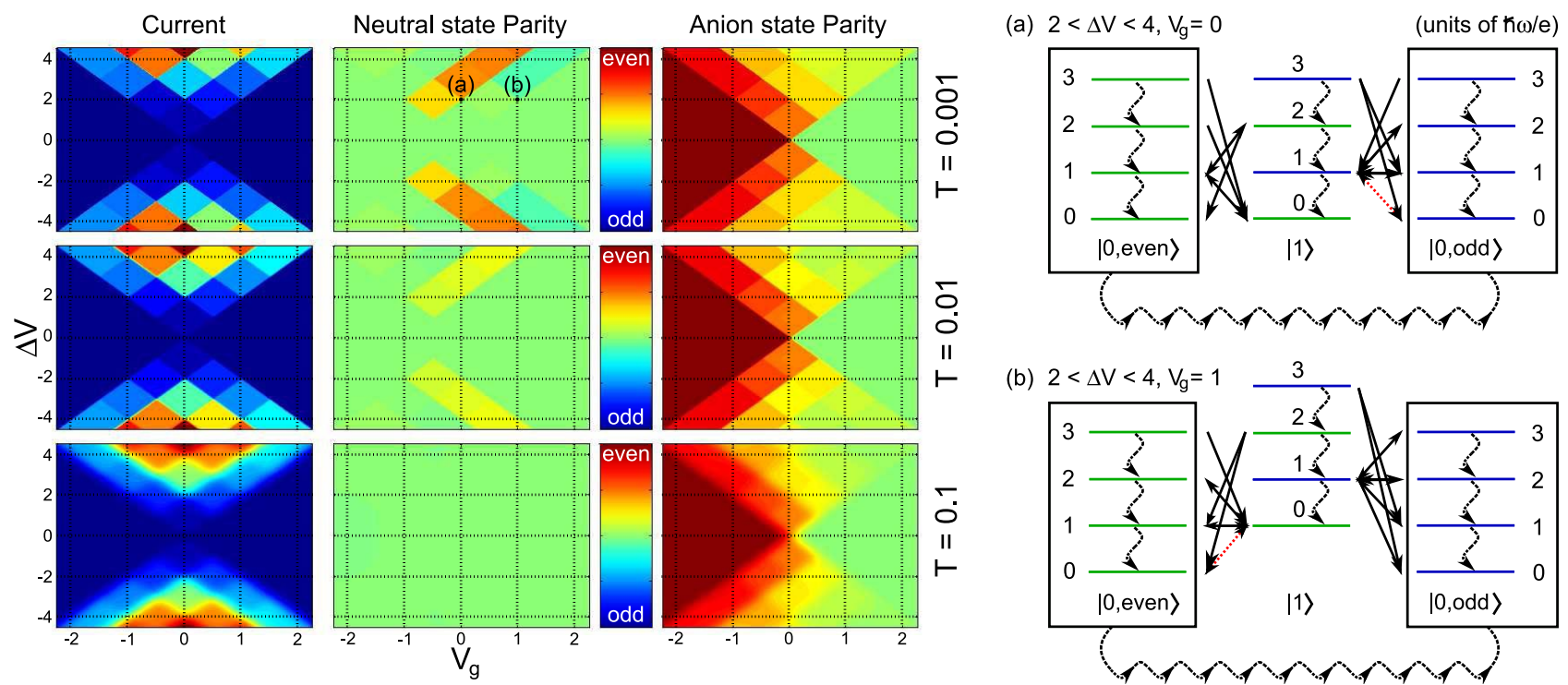

FIG. 2 (color online). Left-hand side: onset of the dynamical symmetry breaking with decreasing temperature (in units of $\hbar \omega_{0} / k_{B}$ ) from bottom to top. The columns show the absolute value of the stationary current and the parity of the neutral and anionic state as a function of the gate voltage $V_{g}$ and the bias $\Delta V$ across the molecular junction, both in units of $\hbar \omega_{0} / e$. The other parameters are $\gamma=0.01 \omega_{0}, \Gamma_{L, R}=0.1 \omega_{0}, \lambda=3 \sqrt{\hbar / m \omega_{0}}$. Right-hand side: rates scheme at the even (a) and odd (b) transition points [corresponding to the points marked by (a) and (b) in the upper middle panel of the figure to the left]. The horizontal lines mark the electromechanical states of the neutral and anionic configuration with green (blue) color representing the even (odd) parity. Straight arrows represent transitions due to the coupling to the leads: the rate is qualitatively expressed by the thickness of the line. Dashed arrows show transitions lifting the blocking character of the ground states and are crucial for the understanding of the symmetry breaking transition. Wavy arrows represent the effect of the phononic bath. 
parities of the neutral and anionic state defined as

$$
\begin{aligned}
& P_{0}=\sum_{n=0}^{N}[\langle 0, e, n|\sigma| 0, e, n\rangle-\langle 0, o, n|\sigma| 0, o, n\rangle], \\
& P_{1}=\sum_{n=0}^{\infty}[\langle 1,2 n|\sigma| 1,2 n\rangle-\langle 1,2 n+1|\sigma| 1,2 n+1\rangle],
\end{aligned}
$$

where the even or odd states of the neutral molecule are

$$
|0, e / o, n\rangle=\frac{1}{\sqrt{2}}\left(|n,+\rangle \pm(-1)^{n}|n,-\rangle\right) \text {. }
$$

In the parameter region where the current becomes asymmetric with respect to $V_{g}$, degenerate states are differently populated, i.e., a dynamical symmetry breaking (DSB) in the occupation of the even or odd states occurs. In the regions of defined parity (warm colors for even, cold for odd) the system exhibits spatial coherences in the ( \pm ) basis cf. Eqs. (8) and (9). In contrast, we find that in the even or odd basis coherences are decoupled from populations and vanish for the stationary density matrix. The onset of these even or odd parity regions in the gate-bias voltage plane can be understood in terms of transition rates between the different vibrational states of the molecule (see the right side of Fig. 2). At zero bias, energy conservation prevents any transition from the neutral (even or odd) or anionic ground states to occur. These states are thus blocking states. In particular, for positive gate voltages the molecule is neutral and dephasing ensures equilibration of the even and odd populations of the degenerate ground state $\left(P_{0}=\right.$ $0)$. At negative gate voltages the molecule is charged and dissipation ensures relaxation predominantly to the anionic ground state $\left(P_{1} \approx 1\right)$.

The situation changes for combinations of bias and gate voltages that allow current to flow: the electromechanical system is maintained out of equilibrium by the applied voltage. Now the parity of the anionic state fluctuates due to population of the higher excited states, and the distribution of the populations no longer corresponds to the thermal distribution in equilibrium with the bath.

The sign of the neutral-state parity depends on the particular rate configuration. Though a quantitative prediction relies on the solution for $\sigma^{\text {stat }}$, it is possible to understand the sign of the neutral-state parity with simple arguments. In the right side of Fig. 2 a representation of the electromechanical rates for two specific cases is reported. Despite the complexity of the scheme, only few lines are crucial: these are the two red dashed arrows representing the rates which lift the blocking character of the neutral ground states. In the case (a) the neutral odd ground state can be depopulated resulting in an even parity of the stationary state. In case (b) the neutral even ground state is involved leading to an odd parity of the stationary state. These unblocking rates represent the bottleneck in the DSB and are competing with the dephasing generated by the heat bath (wavy line joining the even and odd sector of the neutral state). The intensity of the unblocking rates depends on the Franck-Condon factors of the vibrational wave functions involved. A comparison with the dephasing rate allows to estimate the transition temperature $T_{\text {tr }}$ as $k_{B} T_{\mathrm{tr}}=\hbar \omega_{0} \Gamma_{\mathrm{ub}} /\left(4 \lambda^{2} \gamma\right)$, where $\Gamma_{\mathrm{ub}}$ is the unblocking rate (i.e., the depopulation rate of the relevant neutral ground state). The even transition (a) involves a larger $\Gamma_{\mathrm{ub}}$ : it is more robust and occurs at higher $T$ (compare first and second panel in the central column of the left side of Fig. 2).

To summarize, we analyzed the dynamics of a molecular junction in the single-electron transport regime. The molecule possesses quasidegenerate vibrational eigenstates in the neutral configuration and no degeneracy in the anionic case. Tunneling processes charging and discharging the molecule preserve the parity of the wave functions. As a consequence, unequal occupation of degenerate molecular neutral states occurs. An explanation of this effect in terms of unblocking rates is given.

We acknowledge financial support by the DFG within the research school GRK 638. We thank, in particular, M. Čížek for inspiring discussions.

[1] A. Aviram and M. A. Ratner, Chem. Phys. Lett. 29, 277 (1974).

[2] M. A. Reed et al., Science 278, 252 (1997).

[3] For a recent overview see, e.g., Introducing Molecular Electronics, edited by G. Cuniberti, G. Fagas, and K. Richter, Lecture Notes in Physics (Springer, New York, 2005).

[4] L. Y. Gorelik et al., Phys. Rev. Lett. 80, 4526 (1998).

[5] H. Park et al., Nature (London) 407, 57 (2000).

[6] T. Novotný, A. Donarini, and A.-P. Jauho, Phys. Rev. Lett. 90, 256801 (2003).

[7] J. Koch and F. von Oppen, Phys. Rev. Lett. 94, 206804 (2005).

[8] K. C. Nowack and M. Wegewijs, cond-mat/0506552.

[9] M. P. Samanta et al., Phys. Rev. B 53, R7626 (1996).

[10] Z. J. Donhauser et al., Science 292, 2303 (2001).

[11] R. Pati and S. P. Karna, Phys. Rev. B 69, 155419 (2004).

[12] S. Braig and K. Flensberg, Phys. Rev. B 68, 205324 (2003).

[13] A. Troisi, A. Nitzan, and M. Ratner, J. Chem. Phys. 119, 5782 (2003).

[14] A. Mitra, I. Aleiner, and A. J. Millis, Phys. Rev. B 69, 245302 (2004).

[15] M. Čížek, M. Thoss, and W. Domcke, Czech. J. Phys. 55, 189 (2005), and references therein.

[16] T. Dadosh et al., Nature (London) 436, 677 (2005).

[17] L. Venkataraman et al., Nature (London) 442, 904 (2006).

[18] U. Weiss, Quantum Dissipative Systems (World Scientific, Singapore, 1999), 2nd ed. 